\title{
Step-Outs to Stars: Engineering Retention Framework
}

\section{Dr. Nora Honken, University of Cincinnati}

Nora is an Assistant Professor in the Engineering Education Department at The University of Cincinnati. She holds a PhD in Educational Leadership and Organizational Development for the University of Louisville, a MS in Industrial Engineering from Arizona State University and a BS in Industrial Engineering from Virginia Tech. She also has extensive industrial experience.

\section{Dr. Patricia A Ralston, University of Louisville}

Dr. Patricia A. S. Ralston is Professor and Chair of the Department of Engineering Fundamentals at the University of Louisville. She received her B.S., MEng, and PhD degrees in chemical engineering from the University of Louisville. Dr. Ralston teaches undergraduate engineering mathematics and is currently involved in educational research on the effective use of technology in engineering education, the incorporation of critical thinking in undergraduate engineering education, and retention of engineering students. She leads a research group whose goal is to foster active interdisciplinary research which investigates learning and motivation and whose findings will inform the development of evidence-based interventions to promote retention and student success in engineering. Her fields of technical expertise include process modeling, simulation, and process control.

\section{Dr. Thomas Tretter, University of Louisville}

Thomas Tretter is professor of science education and director of the Gheens Science Hall \& Rauch Planetarium at the University of Louisville. His scholarship includes collaborative efforts with science and engineering faculty targeting retention of STEM majors in entry-level STEM courses. 


\title{
The Step-outs to Stars Engineering Retention Framework
}

\begin{abstract}
This research paper, grounded in Expectancy-Value Theory, investigates the relationship between interest, first semester GPA and first year retention in engineering. The research resulted in a purposefully parsimonious framework that can be readily implemented to provide useful discrimination for predicting retention, and can be used when investigating other factors related the retention. Quadrants based on interest were created based on results from logistic regression analysis which showed a difference in the probability of retention between students with equal, less, or no interest in engineering compared to other fields, and those with only or more interest in engineering. GPA quadrants were created based on average GPA. The usefulness of the framework is demonstrated by investigating gender as a variable. The analysis showed a difference of retention rate between males and females only in the quadrant with high grades and low interest.
\end{abstract}

\section{Introduction}

Pressures to increase the retention rate of post-secondary students has risen due to state and federal governmental concerns over retention and student debt, and the use of retention and graduation rates to rank institutions of higher learning ${ }^{1}$. As a result, student retention is one of the most studied subjects in higher education ${ }^{2}$. A subset of this literature concentrated on retention of engineering students has also grown, partly due to the national discussion about the potential shortage of engineers in the workforce (see Charlette ${ }^{3}$ and United States Congress Joint Economic Committee ${ }^{4}$ for a discussion of both sides of the debate). Multiple reasons exist for studying engineers as a separate group including: a) the differences in the demands of the engineering curriculum compared to other college majors ${ }^{5}$; b) the types of students who choose to study engineering ${ }^{6,7,8}$; and c) a belief that factors affect engineering students' performance and persistence decisions differently than non-engineering students 9 .

Studies in engineering retention have been influenced by factors from the college retention literature. These factors include pre-entry characteristics such as skills and abilities ${ }^{10,11}$ family background ${ }^{12}$, institutional experience ${ }^{13,14}$, and finances ${ }^{15}$. While research in college retention has focused on integration into the university, research in engineering retention has focused more on integration into the engineering culture ${ }^{16}$.

Factors not related to college retention, but instead related to college major and career choice, have also been investigated in studies of engineering retention. Some studies have focused on why students made the decision to study engineering ${ }^{17,18,19}$, while others have focused on students' decisions to leave engineering ${ }^{20,21}$. Collectively, these studies investigated factors such as the importance of available jobs, good pay, interest in the field, and perceived ability to perform. 
Other factors related to social and psychological factors have also been investigated with respect to retention in engineering or other majors. These factors include things such as briefs on effort and intelligence ${ }^{22,23}$, feeling of belonging ${ }^{24}$ and grit $^{25}$.

Thus the literature is ripe with factors that have been related to retention of engineering students, as well as, general college students. As such, retention of college students in general, and engineering students in particular, is a complex issue with many interrelated factors that affect students differently and are not easily measurable for all students. This study very intentionally sought a parsimonious, simple model that could be very readily implemented. Our purpose was to explore if such a simple model might provide useful discrimination for predicting retention, and thereby potentially serve as an actionable framework for designing and implementing retention strategies. We also were interested in exploring if investigating other factors within the framework might help explain some conflicting results within the engineering retention literature.

\section{Theoretical Framework}

Many of the studies on engineering retention are framed through the lens of college retention. Although there is value in that perspective, this study was framed in expectancy value theory ${ }^{26}$, a theory used in the college major and career choice literature ${ }^{27,28}$. Expectancy value theory, a motivational theory, attempts to explain individuals' choice of behavior based on their expectation of success and the value they place on the task or outcome of the task. The behavior can be related to the decision to work on a task, whether or not to persist at a task, or the amount of effort to invest in a task ${ }^{28}$. Atkinson, who was the first to form a mathematical model including expectancies, values and behaviors, hypothesized that a person's expectancy was based on a motive to find success and a motive to avoid failure ${ }^{29}$.

\section{Expectancy}

Bandura $^{30}$ discussed two types of expectancy: outcome expectancy and efficacy expectancy. Outcome expectancy deals with the belief that a behavior will lead to a desired outcome; for example, one belief might be that "If I study hard, I will get an A in this class." Efficacy expectancy concerns an individual's belief that he or she can be successful in completing a given task, such as the belief that he or she can complete an engineering degree. The current study focused on efficacy expectancy.

An individual's level of expectancy is impacted by his/her competency, self-efficacy, and their perceived level of control over the task ${ }^{31}$. Weiner ${ }^{32}$ states that expectancy will be lower if the individual's perceived ability is low or his/her perceived difficulty of the task is high. He also states that if an individual assumes that conditions will remain the same and that his past success was due to ability, he will anticipate success in another similar task. Since many students measure success by GPA, first semester GPA was used as a measure of expectancy in the current study. Further support for using GPA to measure expectancy is given in the literature review section. 
Value

Value is related to the incentive or gain from doing or completing a task ${ }^{31}$. Eccles and Wigfield ${ }^{31}$ list four components that determine the value of completing a task: intrinsic, utility, attainment, and cost. Intrinsic value is related to the enjoyment a person gets from engaging in the task. The more interested one is in the task, the higher the intrinsic value. Utility value is based on the contribution the activity makes toward meeting a long-term goal, such as getting a degree. Attainment value includes the benefits gained from completing the task, such as finding a high paying job. Attainment value can also be related to less concrete results such as confirming an aspect of one's self-schema ${ }^{16}$, or the relevance of engaging in the task. Finally, cost value includes the effort needed to engage in the task, as well as the inability to do other tasks, and emotional costs such as anxiety and fear. The current study used interest in engineering as a measure of intrinsic value. The study was not concerned with why the student was interested in engineering, only how their interest in engineering compared to interest in other fields. Further support for using interest as a measure of value is given in the literature review section.

\section{Related Literature}

Why Students Choose to Study Engineering

A number of survey based studies have been conducted to determine why students choose to study engineering. Some have investigated solely engineering students ${ }^{17}$, while others also included science, technology and math majors ${ }^{19,33}$. Conclusions from these studies were dependent upon the design of the survey (wording of the questions and answer options) and the population sampled. For example, surveys that allow multiple responses, rated responses or open responses may come to different conclusions on which factors influenced students to study or leave engineering ${ }^{34}$.

Harris International conducted a national online survey of college students currently pursuing engineering, as well as science, technology, and math fields ${ }^{19}$. Results of the survey showed $86 \%$ of the students were motivated to choose their major based on the belief they could get a good salary; $68 \%$ were motivated by intellectual stimulation and challenges; and $66 \%$ were motivated by job potential.

The same three factors - job availability, good pay, and interest - were cited in published studies at University of Louisville ${ }^{17}$ and Arizona State University ${ }^{33}$. In the Arizona State University study, students were asked why they were interested in engineering or applied science, and were given seven responses to rank in order of importance. Of their top three responses, 79\% selected Potential good salary, 72\% selected Interesting work, and 63\% selected Many job opportunities. In a University of Louisville study ${ }^{17}$, students in the 2011 freshman cohort were asked to respond to nine factors and rank the top three they considered when determining what career to pursue. Holds my interest, was chosen by the highest percentage of students as their top factor (34\%) and was selected by the highest percentage of students in their top three factors (64\%). The next highest response was That I feel confident jobs will be available when I graduate, which was selected as the top factor by $21 \%$ of the students and in 
the top three by $56 \%$. The final response that was selected in the top three by over half of the students was That pays well.

In the University of Louisville ${ }^{17}$ study students were also asked "Why did you chose to study engineering?" The responses included good jobs and good pay, but also included Good at math and science and sources of influence such as parents or teachers encouragement. Over $90 \%$ of the students selected Good in math and science as one of potentially multiple responses. Clearly, the overwhelming majority of engineering students think they are good in the topics that are the foundation of an engineering education. Thus one can conclude they expected to be successful when they choose to study engineering.

Why Students Left Engineering

Other studies have gathered data from students who started in engineering, but subsequently left. A well-referenced multi-institutional ethnographic study ${ }^{21}$ investigated why students who were expected to be successful based on their SAT math scores switched from engineering while others did not. Lack or loss of interest in engineering was one of the top contributing factors to students' decisions to switch out of engineering. A male student who switched out of engineering pointed out the importance of having interest in engineering: "You have to have the interest and the desire. I don't think the problem is preparation. I think its more interest." (p. 179). Other top factors cited in the study related to difficulty of the curriculum, poor teaching and advising, and loss of confidence due to poor grades.

Loss of interest in engineering was also documented as a major cause for leaving engineering in a study that analyzed survey results from students who transferred out of engineering at the University of Pittsburgh ${ }^{35}$. Of the 115 freshmen who completed the survey, $72 \%$ selected Lost interest/developed new interest as a factor in their decision to leave engineering. Additionally, 66\% selected Came to dislike engineering/studying engineering, and $25 \%$ cited Academic problems.

Seymour and Hewitt ${ }^{21}$ reported that $25 \%$ of the students who switched from engineering cited poor grades as a factor in their decision to switch and $40 \%$ cited it as a concern. Shuman, et al. ${ }^{35}$ showed that students on academic probation accounted for half the students who left engineering. Other research has shown a statistically significant difference in the GPA for students who remained in engineering versus those who left the university or switched to another major $^{36,37,38,39}$. Hartman and Hartman ${ }^{13}$ found significant difference in average GPA for males who left engineering and those who stayed, but there was no significant difference for females.

For some students, grades help define their self-worth and a drop in grades can be devastating and can lead students to switch out of challenging disciplines such as engineering ${ }^{21}$. GPA is also important to students due to university-established GPA requirements for scholarships and academic probation.

Based on an unpublished study at the University of Louisville ${ }^{40}$, students start engineering school with expectations of earning high GPAs. In that study, during the first week of class students in the 2012 freshman cohort were asked on a survey what they thought the chances were they would receive a GPA of 3.5 or above and $56 \%$ answered Very good or Good. 
When asked the chances they would receive a 3.0 or above, $86 \%$ of the students responded Very good or Good. This expectation is most likely based on the GPAs they earned in high school (38\% of this cohort had a high school GPA of 4.0 or above). At the end of first semester only $26 \%$ of the cohort had a GPA of 3.5 or above and $44 \%$ had a GPA of 3.0 or above.

Justification of Variables Used to Create the Step Out to Stars Framework

The review of literature presented here shows interest in engineering is a key reason that students choose to study engineering, while loss of interest is also a key reason students leave engineering. Since the other top indicators of value explored in the literature, job outlook or salary projections for engineers, are not mentioned in studies investigating why students leave engineering, interest is a logical measure of value to use when looking at engineering students' decisions to leave or to continue to study engineering. In fact, the justification for considering interest in engineering as a key factor in engineering retention is so strong, we argue studies of retention in engineering should systematically include an interest variable in their models. How individuals develop interest is a complex issue due to the many interrelated factors that can impact an individual's level of interest in a field, and the variety of ways factors impact individuals differently. The current study was only concerned with the level of students' interest in engineering compared with interest in other fields, not the source of their initial interest or source of their decrease in interest.

The use of GPA to measure expectancy is supported by the research discussed above which demonstrated that many students choose engineering as a field of study due to the belief they are good in math and science and the belief they will perform well in engineering, which is most likely due to receiving high grades in the past. The loss of confidence students experience when their GPA drops ${ }^{21}$ also supports using GPA as a measure of expectancy.

Given that both interest and GPA performance have been repeatedly established as important factors in students' decisions when both deciding to study engineering and deciding to leave engineering, this study investigated at what level of interest and GPA performance the likelihood of a student being retained in engineering changed. This knowledge was then used to create a framework that can be used to predict retention, as well as, serve as an analytic mechanism to investigate other variables' effects on retention.

\section{Research Methods}

Participants

The participants in this study were all first-time, full-time students in fall of 2012 at one large metropolitan research institution. The freshman cohort consisted of 430 students. Data from 352 students ( $82 \%$ of the cohort) were used in analysis to determine the threshold value for interest. The ethnic and gender distributions of the participants were similar to the entire 2012 cohort and other recent cohorts at the same university (see Table 1). The sample was less ethnically diverse and had a higher percentage of females than the national population of engineering students ${ }^{41}$. 
Table 1

Ethnic and Gender Distribution of Participants Compared to the Cohort

\begin{tabular}{|c|c|c|}
\hline & In the cohort & $\begin{array}{c}\text { Included in } \\
\text { analysis on interest }\end{array}$ \\
\hline Females & $93 \quad(22 \%)$ & $79 \quad(22 \%)$ \\
\hline Males & $337(78 \%)$ & $273(78 \%)$ \\
\hline Caucasians & $365(85 \%)$ & $(85 \%)$ \\
\hline Asians & $17 \quad(4 \%)$ & $(3 \%)$ \\
\hline Hispanic/Latino & $(4 \%)$ & $(4 \%)$ \\
\hline African American & $(3 \%)$ & $(3 \%)$ \\
\hline Two or more races & $13(3 \%)$ & $(3 \%)$ \\
\hline Nonresident Alien & $2(<1 \%)$ & $2(<1 \%)$ \\
\hline Other & $2(<1 \%)$ & $2(<1 \%)$ \\
\hline First semester GPA & $2.71 \quad(S D=.98)$ & $(S D=.87)$ \\
\hline Composite ACT score & $28.4(S D=3.14)$ & $28.5(S D=3.14)$ \\
\hline
\end{tabular}

\section{Data Collection}

Data used to determine the students' level of interest in engineering were taken from a survey given during week 13 of the fall 2012 semester. The survey was administered by employees in the Office of Institutional Effectiveness and completed by students in Introduction to Engineering, a required course for all freshman engineering students. No rewards or class credit were given for completing the survey, however time was allotted during class. The survey took approximately 10 minutes to complete and included questions on a variety of topics. A similar survey was administered during week one of the semester (week 1 survey). Responses on this survey were used in post hoc analysis to determine if students' level of interest changed throughout the semester.

Measures

Of the three top factors students choose to study engineering (job availability, good pay, and interest) interest in engineering is the only factor that was also given for a reason to leave engineering. This study was interested in the student's interest in engineering compared to other fields, so the following question was asked and students were able to select only one answer. 
Which of the following statements best describes your interest in engineering?

1. Very low interest--I'm not interested in engineering; I chose engineering for reasons other than interest.

2. Low interest-I have an interest in engineering but stronger interest in another field(s).

3. Medium interest--I am interested in engineering and equally interested in another field(s).

4. High interest--I am very interested in engineering, but also think I could be happy in another field.

5. Very high interest--I am so interested in engineering that I could not imagine myself studying anything else.

Students' first semester GPA was used as a measure of expectancy. First semester GPA for engineering students at the institution studied generally includes grades on the following courses: Engineering Calculus, Chemistry, and Introduction to Engineering. Students' retention in engineering status after one year and their first semester GPAs were extracted from student records maintained by the university. A student was considered retained in engineering if in the fall of their second year they were enrolled in the university and their academic unit was engineering.

Missing Data

The National Center of Education Statistics (NCES) requires missing data analysis on their studies with response rates lower than $85 \%{ }^{42}$. Since the response rate to the survey was $82 \%$, we investigated the ACT scores and first semester GPA of students who did not participate in the study compared to those who did participate. The non-participants did have statistically significant lower ACT math and science scores and first semester GPA compared to the participant group. However, when combining both the participants and nonparticipants into one group and comparing this overall full cohort with the subset of participants, there was no statistically significant difference in any of the ACT subject scores or first semester GPA between the participants and the entire cohort.

\section{Data Analysis}

The interest level and student performance and retention data were analyzed using logistic regression in SPSS version 21. Logistic regression can be used to predict a dichotomous outcome based on independent variables, or as in this study, to understand the relationship between independent variables and a dichotomous variable for the purpose of building or validating a theory ${ }^{43}$. The outcome variable STATUS was coded to " 1 " if the student was retained in engineering at the same university (their academic unit fall 2013 was engineering), and it was coded to "0" if the student left the university or switched to another academic unit within the university. 
The response to the question on interest in engineering (INTEREST) could have been treated as a continuous or categorical variable. Since the objective of the analysis was to determine if there was a difference in the likelihood of retention between specific levels of interest, the variable was treated as a categorical variable. Due to the threat to statistical validity from scarcity of data for the responses of Very low and Low, the responses of Very low, Low and Medium were condensed into one category (INTEREST1). The responses of High were entered as variable INTEREST2 and the responses of Very High were used as the reference category. The equation resulting from logistic regression had the following form:

$$
\begin{aligned}
\ln \left(\frac{p}{1-p}\right)= & \beta_{\text {constant }}+\beta_{F A L L G P A} \text { FallGPA } \\
& +\beta_{\text {INTEREST }(1)} \operatorname{INTEREST}(1) \\
& +\beta_{\text {INTEREST(2) }} \operatorname{INTEREST}(2)
\end{aligned}
$$

Results of the Wald test were used to determine the significance of GPA and INTEREST using .05 as the criteria of statistical significance. The odds ratios for each independent variable were used to measure effect size.

Threats to Validity

The results of this study must be viewed in light of the potential threats to validity. As with all studies based on survey data, we do not know how accurately participants' responses represented their true interest level in engineering. When analysis was performed by gender the sample size, although appropriate for the analysis, was rather small. Finally, all the respondents in this study were from one cohort at the same university and were less ethnically diverse than the national population of engineering students. This might lead to a problem with external validity if the results were applied to a group of students that differ greatly from the sample used in this study.

\section{Results}

Table 2 summarizes the variables used in the logistic regression analysis and includes the variable name, variable type, potential values, and the sample size for the categorical data. The average GPA for the participants was $2.71(S D=.98)$. If INTEREST was treated as a continuous variable using all five values of interest, the correlation between the INTEREST and GPA (.181) was statistically significant, but lower than the level $(\geq .8)$ at which collinearity poses a statistical threat to validity ${ }^{43}$. 
Table 2

Information on Variables for Research Question 1 Analyses

\begin{tabular}{lclrr}
\hline Variable & & Type & Potential values & $n$ \\
\hline $\begin{array}{l}\text { Retention status } \\
\text { (outcome variable) }\end{array}$ & (STATUS) & Categorical & $0=$ No & 92 \\
& & $1=$ Yes & 260 \\
Fall 2012 GPA & (GPA) & Continuous & 0 to 4.0 & \\
Interest in engineering & (INTEREST1) & Categorical & $1=$ Very low, Low & 74 \\
& & & and Medium & \\
& (INTEREST2) & $2=$ High & 197 \\
& Reference category & $3=$ Very high & 81 \\
\hline
\end{tabular}

Table 3 contains the coefficient estimates and the Wald statistic for each variable along with the odds ratio and upper and lower confidence intervals of the odds ratio. These results confirmed prior research that both interest in engineering and academic performance were significant variables in predicting the likelihood of a student being retained after one year $(\mathrm{p}<$ .001). The lack of significance of INTEREST2 indicated that there is no significant difference in the likelihood of a student with very high or high interest being retained after one year, given the same GPA.

Based on the odds ratios a student was 4.6 times more likely to be retained than a student with a one point lower GPA, given the same interest level. Also a person with very high interested was about $7.7(1 / .131)$ times more likely to be retained than a student with equal or more interest in a field other than engineering, given the same GPA.

Table 3

Results from Logistic Regression Analysis

\begin{tabular}{lrrrrrrr}
\hline & $B$ & S.E. & Wald & df & Sig. & $\begin{array}{c}\text { Odds } \\
\text { Ratio }\end{array}$ & \multicolumn{1}{c}{$\begin{array}{l}\text { 95\% C.I. for } \\
\text { Odds Ratio }\end{array}$} \\
\hline F12_GPA & 1.530 & .204 & 56.129 & 1 & $<.001$ & 4.618 & {$[3.095,6.891]$} \\
INTEREST & & & 29.073 & 2 & $<.001$ & & \\
INTEREST1 & -2.034 & .449 & 20.546 & 1 & $<.001$ & .131 & {$[.054, .315]$} \\
INTEREST2 & -.391 & .410 & .910 & 1 & .340 & .676 & {$[.303,1.511]$} \\
\hline
\end{tabular}

Although the purpose of the analysis was exploratory and the main emphasis of the analysis was to investigate the significance of the odds ratios, the fit statistics for the model still warranted review. The chi-square test comparing the fit between the model with no predictors and the hypothesized model indicated that INTEREST and GPA help the model fit the data significantly better than no predictors, $\chi^{2}(3)=122.22, p<.001$. Cox and Snell $\mathrm{R}^{2}$ was .293 and 
Nagelkerke $\mathrm{R}^{2}$ was .429 , which give a sense of the magnitude of percent variance explained by these predictors. Overall the model correctly predicted the status of $83 \%$ of the students, which was better than the null model that correctly predicted status for $74 \%$ of the students. The model correctly predicted $94 \%$ of the students who were retained, but only $52 \%$ of the students who did not continue to study engineering. The lower percentage predicted for non-retained students is most likely driven by the substantially smaller sample size for that group.

Outlier analysis was performed and the model was rerun without suspected outliers. The results continued to show a significant difference in the probability of being retained between students with very high interest and very low, low or medium interest, and no difference between students with very high interest and high interest.

\section{Step-outs to Stars Engineering Retention Framework}

Based on the results of the logistic regression analysis, an engineering retention framework was constructed. The framework consists of a 2 by 2 array producing four quadrants based on first semester GPA and interest in engineering after one semester. The two classifications of interest (rows in the framework) are "equally or more interested in a field other than engineering" (low) and "more interested in engineering than any other field" (high). These interest categories were chosen as meaningfully distinct from each other since the analyses showed no difference in probability of being retained for students with high or very high interest, but a significant difference between very high and very low, low and medium interest.

Using a mean split approach, the two (column) classifications of GPA are below average (low) and above average (high). To establish the rationale for this approach of generating useful GPA categories for this framework, we first examined the logistic regression model to confirm that there was a significant relationship between GPA and the likelihood of being retained. However, since GPA was treated as a continuous variable in the logistic regression model, those results did not give a clear GPA threshold value for meaningfully distinguishing between high and low performance for the engineering retention framework. We considered using 3.0 based on the use of a 3.0 as a minimum GPA to maintain many scholarships ${ }^{15,44}$, and the use of 3.0 as a interview minimum by many employers ${ }^{45}$. We also considered using a unique value for each student based on their own definition of the GPA they considered a success, but this was ruled out since it would most likely not be available to other colleges using the framework. We considered using a 2.7 (B-) since this is the lowest value that still represents a B average. In the end for this analysis, we decided to use the cohort average (2.71) which was very close to a B-, although other cut-off values might be meaningful for other users of the framework. The quadrants produce a characterization of students as Stars (high interest, high GPA), Searchers (high GPA, low interest), Strugglers (high interest, low GPA) and Step-outs (low interest, low GPA) - see Figure 1. The labels given to students who fall in each quadrant were generated to capture the essential feature of each particular combination of interest and performance. 
Figure 1 shows the number and percentage of students from the 2012 cohort in each of the four quadrants of the retention framework: Stars, Searchers, Strugglers, and Step-outs. Also shown are the percentage of the students within the quadrant who were retained, switched out of engineering or left the university.

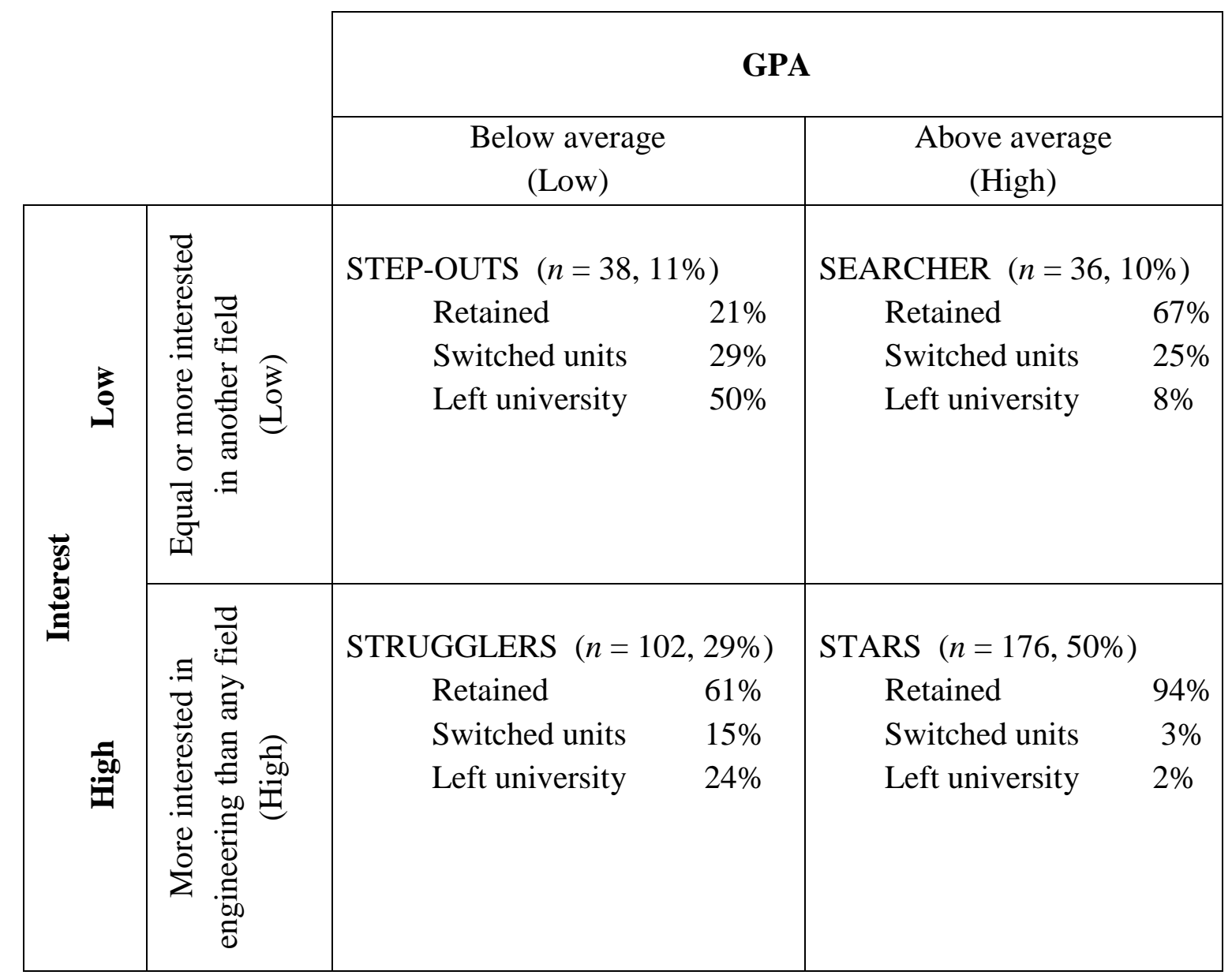

Figure 1. Step-outs to Stars engineering retention framework

Quadrants in the Framework

Stars. In the framework, the students in the quadrant with high GPA and high interest were named the Stars. Based on the odds ratio from the model, the Stars would be expected to have the highest retention rate. The actual first year retention rate for the students from the 2012 cohort in this quadrant was $94 \%$, which was the highest of all the quadrants. Based on the simplicity of the framework and the exclusion of factors such as finances and commitment to the university, the accuracy of prediction in this quadrant was high. It was not surprising that these students had the highest retention rate as they seem to have found a good fit between interest and ability. Fifty percent of the students in the 2012 cohort were in this quadrant. 
At the start of the semester, $21 \%$ of the Stars indicated they had very high interest in engineering and the percentage increased to $27 \%$ at the end of the semester. Post hoc analysis, using the chi square goodness of fit test and all response categories to the question on interest, showed a significant positive change in interest for the Stars $\left(\chi^{2}(3)=12.12, p=.007\right)$ from the first week of the semester to week 13.

Step-outs. The students in the opposite quadrant with low GPA and low interest, referred to as the Step-outs, would be predicted to have the lowest retention rate based on the logistic regression results. This group represented $11 \%$ of the 2012 cohort and their retention rate of $21 \%$ was the lowest of any quadrant. Half of the Step-outs left the university and $29 \%$ switched to another unit.

The Step-outs had a significant change in their responses to the interest in engineering question from the week 1 survey to the week 13 survey $\left(\chi^{2}(3)=429.27, p<.001\right)$. On the week 1 survey, $76 \%$ of the Step-outs selected a higher interest category than they did on the week 13 survey. It is unknown if the students in this quadrant chose engineering as a major not knowing much about the field or the curriculum, if they lost interest due to their lackluster performance, or if their interest changed for some other reason. Future research could be conducted to determine the reasons that these students chose to study engineering and if better career advising could have helped them make a better decision that was more related to their interest and abilities. It would also be interesting to determine if their poor performance affected their interest level in engineering.

Searchers. Based on the logistic regression model, the two remaining quadrants, one characterized by high GPA and low interest (Searchers) and the other characterized by low GPA and high interest (Strugglers), would be the hardest to predict since the variables suggest opposite relationships with the likelihood of being retained in engineering. The Searchers (10\% of students) had the second-highest retention rate and the highest rate of switching to other units. Based on their responses to the interest question of the week 1-survey, 57\% of the Searchers indicated a higher level of interest in engineering at the start of the semester than in week 13 of the same semester. Their shift in interest was significant $\left(\chi^{2}(3)=59.96, p<.001\right)$.

The Searchers have the ability to do above-average work in engineering, but might not be interested enough to continue to study engineering. The Searchers would likely benefit from career advising or activities that help them maintain interest in engineering. Future research could investigate if students from this group switch units later in their studies. Another interesting study would be to investigate the reasons these students indicated lower interest levels at the end of the semester than at the beginning, considering they were performing aboveaverage.

Strugglers. The third highest retention rate was for students with low GPA and high interest. The Strugglers represented $29 \%$ of the 2012 cohort. The percent of students in this 
group who left the university was less than half the percent of Step-outs who left (low interest, low GPA), even though their average GPA was not statistically different, $t(138)=1.365, p=$ .171. The percent of this group that switched to another unit was $46 \%$ less than the percent of the Searchers who switched units.

At the beginning of the semester, $30 \%$ of the Strugglers indicated they had very high interest in engineering. At the end of the semester, the percentage had increased slightly to $33 \%$, which was higher than the percentage of Stars that had indicated they had very high interest. The data did not show a significant shift in interest between the beginning and the end of the semester for the Strugglers $\left(\chi^{2}(3)=4.33, p=.228\right)$.

The Strugglers may benefit the most from tutoring and mentoring. Future research should investigate Strugglers to determine what factors contributed to their low performance. Further, it should be studied as to whether their high interest will enable them to persist and be successful, or whether they ultimately cannot complete their studies.

Example Analyzing Gender within the Step-outs to Stars Engineering Retention Framework

To demonstrate the benefit of characterizing students using the Step-outs to Stars Engineering Retention Framework, we investigated the variable of gender within the framework. Figure 2 shows for each quadrant of the framework the number and percentage of males and females who were retained in engineering, switched out of engineering but remained at the university, or left the university. The percentages of females in each quadrant were representative of the percentage of females who participated in the study (22\%), except in the Searcher quadrant where $36 \%$ percent of the Searchers were female.

The female Stars had the same retention rate as the male Stars and the female Strugglers and Step-outs had similar retention rates as their male counterparts (caution there were only eight female Step-outs). This was not true for the Searchers where the retention rate for males was $32 \%$ higher than for females.

Although caution must be taken when viewing these results due to the low number of female students in certain quadrants, placing the variable gender within the framework appears to indicate that female students with equal or more interest in a field other than engineering (low interest) and above average GPAs (high GPA) are more likely to switch out of engineering than males in the same quadrant, whereas the other 3 quadrants had similar percentages of retention of both males and females. If this sample is representative of other cohorts, this could help explain why some cohorts have lower retention rates for female students; it appears that female "interest" among high-performing students is a key difference between retention for males and females. These results could help identify areas to target to help increase female retention. 


\begin{tabular}{|c|c|c|c|c|c|}
\hline STEP-OUTS & M (30) & $\mathrm{F}(8)$ & SEARCHER & M (23) & F (13) \\
\hline Retained & $20 \%$ & $25 \%$ & Retained & $78 \%$ & $46 \%$ \\
\hline Switched units & $30 \%$ & $25 \%$ & Switched units & $13 \%$ & $46 \%$ \\
\hline Left university & $50 \%$ & $50 \%$ & Left university & $9 \%$ & $8 \%$ \\
\hline \multirow[t]{2}{*}{ STRUGGLERS } & & & \multicolumn{3}{|l|}{ STARS } \\
\hline & M (79) & $\mathrm{F}(23)$ & & M (141) & $\mathrm{F}(35)$ \\
\hline Retained & $63 \%$ & $52 \%$ & Retained & $94 \%$ & $94 \%$ \\
\hline Switched units & $14 \%$ & $17 \%$ & Switched units & $3 \%$ & $6 \%$ \\
\hline Left university & $23 \%$ & $30 \%$ & Left university & $3 \%$ & $0 \%$ \\
\hline
\end{tabular}

Figure 2. Step-outs to Stars engineering retention framework for males and females

\section{Conclusion}

The Step-outs to Stars engineering retention framework, is a parsimonious framework based on two relatively simple variables to acquire, early performance and student's interest in engineering compared to other fields. We propose it can be a valuable framework for other studies in engineering retention and possibly other demanding fields of study. Since academic performance and interest are the top reasons students switch out of engineering ${ }^{20,21}$, investigation of other factors related to retention could be viewed relative to the student's characterization in this framework. For example, by viewing gender with respect to the framework, it quickly became obvious that in this cohort a disproportionate number of the Searchers were female and the discrepancy in the retention rate between males and females was due to the number of students in the Searchers quadrant.

The interest/GPA characterization appears to be a useful grain size - not excessive number of categories, but nevertheless meaningfully distinct - to situate explorations of other variables in a more nuanced manner that permits more systematic and targeted decisions to be made to help individual students be more successful. Viewing other factors through the lens of this framework might help explain some of the inconsistencies found in the previous research on engineering retention (see Zhang et al. ${ }^{46}$ for an example). Examples of factors that could be investigated with respect to the framework include: ethnicity, self-esteem, social integration into engineering, what influenced students to study engineering, time spent on classwork and studying, student participation in high school activities related to engineering, when students became interested in engineering, and beliefs on effort and intelligence. By investigating these factors within the framework, researchers could start to develop profiles of the students in each 
quadrant. Though these profiles might vary with institution, they might enable advisors and counselors to better understand their student populations.

As colleges of engineering work to increase their retention and graduation rates, and government agencies, universities, corporations, and other organizations work to ensure an adequate supply of engineers to meet the demands of the workforce, these refined quadrants could then be used to help determine the best allocation of resources targeted to improving retention. For example, resources could be directed towards the Strugglers by offering tutoring or supplemental instruction since low GPA seems to be the variable holding them back. Based on the participants in this study, this could impact around $30 \%$ of the students. Resources could be directed toward the Stars, about $50 \%$ of the students in this study, by creating more opportunities for them to develop their interest and continue to excel, but that decision would have to be made with the foreknowledge that these students are very likely to be retained anyway, and so expenditure of resources on this group would be for other purposes, such as quality or depth of experiences rather than for retention purposes. Or resources could be directed toward the Step-outs and the Searchers, each representing around $10 \%$, by investigating ways to increase interest in engineering or providing more opportunities to learn about engineering and the engineering curriculum before deciding to study engineering.

If characteristics of students who fall into each quadrant were more clearly identified, the framework could also be of value to students considering engineering as a college major. The more students know about the skills and personal characteristics of successful engineering students, the better equipped they will be to make their college major choice.

\section{References}

[1] DeAngelo, F., Fanke, R., Hurtado, S., \& Pryor, J. (2011). Completing college: Assessing graduation rates at four-year institutions. Los Angela's, CA: Higher Education Research Initiative.

[2] Tinto, V. (2006-2007). Research and practice of student retention: What next. Journal of College Retention, $8(1), 1-19$.

[3] Charette, R. N. (2013). The STEM Crisis is a Myth, IEEE Spectrum, Retrieved February 28, 2014 from http://spectrum.ieee.org/at-work/education/the-stem-crisis-is-a-myth

[4] United States Congress Joint Ecomonics Committee. (2012). STEM Education: Preparing for Jobs of the Future. Retrieved on March 3, 2014 from http://www.jec.senate.gov/public/index.cfm?a=Files.Serve\&File id=6aaa7e1f-9586-47be-82e7$\underline{326 f 47658320 .}$.

[5] National Society of Student Engagement. (2011). Fostering student engagement campuswide-annual results 2011. Bloomington, IN: Indiana University Center for Postsecondary Research.

[6] Boylan, M. (n.d.). Characteristics of first-time freshmen in 4-year institutions intending to major in engineering and computer science: An analysis of survey data collected by the higher education research institute (HERI): Center for Advancement of Scholarship in Engineering Education.

[7] National Center for Science and Engineering Statitistics. (2012). Science and engineering indicators 2012. Retrieved May 6, 2013, from http://www.nsf.gov/statistics/seind12/c2/c2s2.htm\#s2 
[8] Zhang, G., Carter, R., Thorndyke, B., Anderson, T. J., \& Ohland, M. W. (2003). A comparison of demographic factors and academic performance between students graduated in engineering and other disciplines. Paper presented at the American Society of Engineering Education Southeast Division, Nashville, TN.

[9] Veenstra, C. P., Dey, E. L., \& Herrin, G. D. (2008). Is modeling of freshman engineering success different for modeling of non-engineering success? Journal of Engineering Education, 97, 467-479.

[10] Burtner, J. (2004). Critical-to-quality factors associated with engineering student persistence: The influence of freshman attitudes. Paper presented at the Frontiers in Education, 2004. FIE 2004. 34th Annual.

[11] Mendez, G., Buskirk, T. D., Lohr, S., \& Haag, S. (2008). Factors associated with persistence in science and engineering majors: An exploratory study using classification trees and random forests. Journal of Engineering Education, 97, 57-70.

[12] Eris, O., Chachra, D., Chen, H. L., Sheppard, S., Ludlow, L., Rosca, C., . . Toye, G. (2010). Outcomes of a longitudinal administration of the persistence in engineering survey. Journal of Engineering Education, 99, 371-395.

[13] Hartman, H., \& Hartman, M. (2006). Leaving engineering: Lessons from Rowan University's College of Engineering. Journal of Engineering Education, 95, 49-61.

[14] Marra, R. M., Shen, D., Rodgers, K. A., \& Bogue, B. (2009). Leaving engineering: A multi-year single institution study. Paper presented at the Annual Meeting of the American Educational Research Association, San Diego, CA.

[15] Zhang, G., Min, Y., Frillman, S. A., Anderson, T. J., \& Ohland, M. W. (2006). Students strategies for protecting merit-based scholarships: Grades, courseload, and major choice. Paper presented at the 36th ASEE/IEEE Frontiers in Education Conference, San Diego, CA.

[16] Matusovich, H. M., Streveller, R. A., \& Miller, R. L. (2010). Why do students choose engineering? A qualitative, longitudinal investigation of student's motivational values. Journal of Engineering Education, 99, 289-302.

[17] Honken, N. B., \& Ralston, P. (2013). Freshman engineering retention: A holistic look. Journal of STEM Education: Innovations and Research, 14(2), 29-37.

[18] McIlwee, J. S., \& Robinson, J. G. (1992). Women in engineering: Gender, power, and workplace culture. Albany: State University of New York.

[19] Microsoft. (2011). Microsoft releases national survey findings on how to inspire the next generation of doctors, scientists, software developers and engineering. Retrieved from http://www.microsoft.com/Presspass/press/2011/sep11/09-07MSSTEMSurveyPR.mspx

[20] Besterfeld-Sacre, M., Atman, C. J., \& Shuman, L. J. (1997). Characteristics of freshman engineering students: Models for determining student attrition in engineering. Journal of Engineering Education, 86, 139-149.

[21] Seymour, E., \& Hewitt, N. M. (1997). Talking about leaving: Why undergraduates leave the sciences. U.S.A.: Westview Press.

[22] Blackwell, L. S., Trzesniewski, K. H., \& Dweck, C. S. (2007). Implicit theories of intelligence predict achievement across an adolescent transition: A longitudinal study and an intervention. Child development, 78(1), 246-263.

[23] Honken, N. B., Synder, K. E. \& Ralston, P. A. (2014, June). Exploring Engineering Students' Beliefs on Effort and Intelligence. Paper presented at the 2014 121th ASEE Annual Conference, Indianapolis, IN.

[24] O'Keeffe, P. (2013). A sense of belonging: Improving student retention. College Student Journal, 47(4), 605613.

[25] Duckworth, A. L., Peterson, C., Matthews, M. D., \& Kelly, D. R. (2007). Grit: perseverance and passion for long-term goals. Journal of personality and social psychology, 92(6), 1087.

[26] Atkinson, J. W. (1964). An introduction to motivation. Oxford England: Van Nostrand.

[27] Feather, N. (1982). Expectations and actions: Expectancy-value models in psychology: Lawrence Erlbaum Assoc Inc. 
[28] Wigfield, A., \& Eccles, J. S. (2000). Expectancy-value theory of achievement motivation. Contemporary Educational Psychology, 25(1), 68-81.

[29] Wigfield, A., Tonks, S., \& Klauda, S. L. (2009). Expectancy-value theory. Handbook of Motivation at School. New York: Routledge.

[30] Bandura, A. (1997). Self-efficacy: The exercise of control. U.S.A.: Worth Publishers.

[31] Eccles, J. S., \& Wigfield, A. (2002). Motivational beliefs, values, and goals. Annual Review of Psychology, 53(1), 109.

[32] Weiner, B. (1976). An attributional approach for educational psychology. Review of Research in Education, 4, 179-209.

[33] Anderson-Rowland, M. R. (1997). Understanding freshman engineering student retention through a survey. Paper presented at the ASS Annual Conference, Milwaukee, WI.

[34] Dillman, D. A., Smyth, J. D., \& Christian, L. M. (2009). Internet, mail, and mixed-mode surveys: The tailored design method (3rd ed.). Hoboken, NJ: John Wiley \& Sons.

[35] Shuman, L. J., Delaney, H., Wolfe, A., Scalise, A., \& Besterfeld-Sacre, M. (1999). Engineering attrition: Student characteristics and educational initiatives. Paper presented at the American Society of Engineering Education Annual Conference and Exposition, Charlotte, NC.

[36] French, B. F., Immekus, J. C., \& Oakes, W. C. (2005). An examination of indicators of engineering students' sucess and persistence. Journal of Engineering Education, 94, 419-425.

[37] Goodman, I., Cunningham, C., Lachapelle, C., Thompson, M., Bittinger, K., Brennan, R., \& Delci, M. (2002). Final report of the women's experiences in college engineering (WECE) project. Cambridge, MA: Goodman Research Group, Inc.

[38] Moses, L., Hall, C., Wuensch, K., DeUrquidi, K., Kauffman, P., Swart, W., . . Dixon, G. (2011). Are math readiness and personality predictive of first-year retention in engineering? The Journal of Psychology, 145(3), 229-245.

[39] Veenstra, C. P., Dey, E. L., \& Herrin, G. D. (2009). A model for freshman engineering retention. In A. S. o. E. Education (Ed.), Advances in Engineering Education (Vol. Winter 2009).

[40] University of Louisville Institutional Effectiveness.[ Pre Engineering Student Survey]. Unpublished raw data.

[41] National Science Foundation. (2013). Women, minorities and persons with disabilities in science and engineering (table 2-9 Undergraduate enrollment in engineering programs by sex, race/ethnicity, citizenship and enrollment status: 1999-2009). In National Science Foundation (Ed.). Arlington, VA.

[42] Chen, X. (2013). STEM attrition: College stduents paths into and out of STEM fields (nces 2014-01). Washington, DC: National Center for Education Statistics, Institute of Education Sciences.

[43] Osborne, J. (2015). Best practices in logistic regression. Thousand Oaks, CA: Sage Publishing, Inc.

[44] Mobley, C., Brawner, C., \& Ohland, W. (2009). The south carolina merit scholarship; strategies used by engineering students to keep their life scholarship. International Journal of Engineering Education, 25, 1249-1256.

[45] National Association of Colleges and Employers. (2010). Job outlook 2011. Bethlehem, PA: National Association of Colleges and Employers.

[46] Zhang, G., Anderson, T. J., Ohland, M. W., \& Thorndyke, B. R. (2004). Identifying factors influencing engineering student graduation: A longitudinal and cross-institutional study. Journal of Engineering Education, 93, 313-320. 